ACCEPTED MANUSCRIPT

\title{
Hot photons and open-circuit voltage in molecular absorbers
}

To cite this article before publication: Branislav Dzurnak et al 2019 Semicond. Sci. Technol. in press https://doi.org/10.1088/1361-6641/ab419f

\section{Manuscript version: Accepted Manuscript}

Accepted Manuscript is "the version of the article accepted for publication including all changes made as a result of the peer review process, and which may also include the addition to the article by IOP Publishing of a header, an article ID, a cover sheet and/or an 'Accepted

Manuscript' watermark, but excluding any other editing, typesetting or other changes made by IOP Publishing and/or its licensors"

This Accepted Manuscript is @ 2019 IOP Publishing Ltd.

During the embargo period (the 12 month period from the publication of the Version of Record of this article), the Accepted Manuscript is fully protected by copyright and cannot be reused or reposted elsewhere.

As the Version of Record of this article is going to be / has been published on a subscription basis, this Accepted Manuscript is available for reuse under a CC BY-NC-ND 3.0 licence after the 12 month embargo period.

After the embargo period, everyone is permitted to use copy and redistribute this article for non-commercial purposes only, provided that they adhere to all the terms of the licence https://creativecommons.org/licences/by-nc-nd/3.0

Although reasonable endeavours have been taken to obtain all necessary permissions from third parties to include their copyrighted content within this article, their full citation and copyright line may not be present in this Accepted Manuscript version. Before using any content from this article, please refer to the Version of Record on IOPscience once published for full citation and copyright details, as permissions will likely be required. All third party content is fully copyright protected, unless specifically stated otherwise in the figure caption in the Version of Record.

View the article online for updates and enhancements. 


\title{
Hot photons and open-circuit voltage in molecular absorbers
}

\author{
Branislav Dzurňák ${ }^{1}$, Tianxian Feng ${ }^{1}$, Lefteris Danos ${ }^{2}$ and Tom Markvart ${ }^{1,3}$ \\ ${ }^{1}$ Centre for Advanced Photovoltaics, Faculty of Electrical Engineering, Czech Technical University, \\ Prague, Czech Republic \\ ${ }^{2}$ Chemistry Department, Lancaster University, Lancaster, United Kingdom \\ ${ }^{3}$ Engineering and the Environment, University of Southampton, Southampton, United Kingdom
}

E-mail: branislav.dzurnak@fel.cvut.cz

Received xxxxxx

Accepted for publication $\mathrm{xxxxx}$

Published xxxxxx

\begin{abstract}
Hot carrier solar cells have attracted interest for many years. Although no working exemplars exist today, the challenges to overcome have become clearer and a substantial research effort has been underway with a focus on inorganic semiconductors, including quantum wells. In this paper we propose a novel strategy to potentially exploit hot photons, based on organic absorbers. Our approach, when combined with photon management structures similar to photonic fluorescent collectors, can potentially enhance the efficiency of complete photovoltaic devices. We present a characterisation method of fluorescent collectors by evaluating the chemical potential and temperature of the emitted fluorescence photon flux. We report on observation of temperatures of the emitted photon flux well above the ambient temperature, indicating the presence of hot photons. We propose a theoretical background to describe how excess thermal energy carried by hot photons can be exploited to increase the chemical potential of the photon flux which is closely related to the open-circuit voltage of the solar cell.
\end{abstract}

Keywords: hot carriers, fluorescence, collectors, solar cells

\section{Introduction}

In conventional solar cells, photons with energies higher than bandgap are absorbed and the excess energy is dissipated as heat. This is one of the factors that limit the conversion efficiency of a solar cell. Hot carrier solar cell is a concept that can exploit the excess energy of these photons. This idea has been introduced by Ross and Nozik [1] for semiconductor systems where the excess energy appears as kinetic energy of these carriers. The term "hot carriers" assumes the presence of equilibrium amongst the carriers but not with the surrounding environment. In a solar cell, the excess energy may then be converted to voltage before the hot system reaches equilibrium with the environment at room temperature. Ross and Nozik predicted a theoretical maximum efficiency of $66 \%$ for one-sun illumination.

The major mechanism of energy loss of hot carriers is thermalisation [1-3]. Thermalisation usually occurs on a very short time scale (ps), much faster than the radiative recombination process. Strategies proposed for efficient extraction therefore consider either a fast mechanism of energy conversion or slowing down the thermalisation process. The main effect responsible for thermalisation losses is heat transfer to the lattice due to phonon interaction [3-5]. In order to slow down this interaction, the formation of a phonon bottleneck has been proposed in devices based on quantum wells [5-10]. Fast extraction of carriers is usually suggested to take place by energy selective contacts, based on 
a very narrow energy range of extraction [11]. Quantum dotsbased tunneling structures have been proposed [5, 8, 12], although a practical demonstration of a realistic design is yet to be achieved [13] and alternative approaches have been considered [14-16] as a way forward.

In this paper, we investigate the promise of organic dyes as absorbers in hot carrier solar cells. Whilst the standard model of molecular spectra is founded on the ambient temperature $[17,18]$, elevated temperatures are also frequently observed [19-21]. By using BASF red dye 305 we report here on the observation of an equilibrium photon flux at a temperature well in excess of the ambient temperature, generated by the edge emission from a fluorescent collector (FC) with an organic dye layer used as an absorber. We analyse the emitted flux by means of fluorescence (FL) spectroscopy and apply the generalised Planck's law to the reabsorbed photon emission from the edge of the collectors, which leads to temperatures above the ambient temperature. We calculate the enhancement in the open-circuit voltage of a potential photovoltaic device based on hot photon reversible energy conversion. We show that the thermodynamic properties of emitted photons serve as an ultimate tool for assessment of performance limits of the absorber, including absorbers based on population of hot carriers.

\section{Temperature and reabsorption of photon flux}

Measurements were made of spectra of photons emitted from the edge of the FC based on a BASF red perylene dye. Spectra with different photon path lengths were obtained by positioning the excitation laser beam at several spots at various distances from the edge of the FC. Emission spectra were collected with an integrating sphere, allowing the evaluation of the absolute photon flux. A schematic of the setup is shown in Fig. 1, detailed description of experiments is included in Appendix. The edge spectra were compared with the front surface spectra obtained from low-concentration $\left(5 \times 10^{-5} \mathrm{M}\right)$ collectors which are considered free from reabsorption and will be taken as the first-generation spectrum.

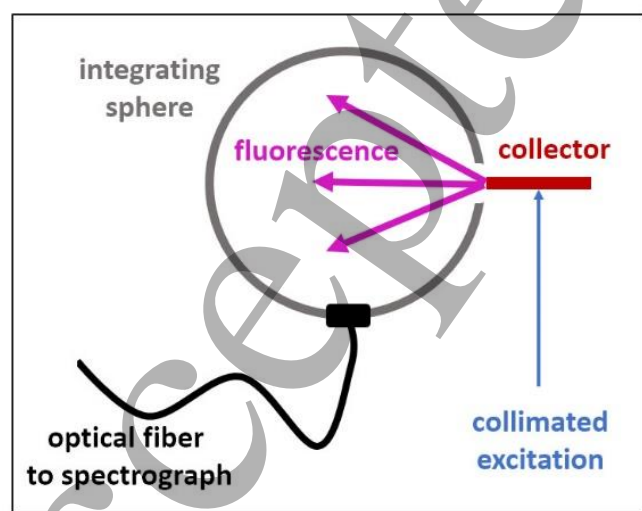

Fig. 1 Schematic of experimental setup for edge FL detection
Because of the long path length through the FC, the FL photons emitted from the edge of the collector are repeatedly reabsorbed and reemitted. The amount of reabsorption depends on the overlap of the absorption with the emission spectra and can be characterized by a probability of reabsorption $r$. The effect of increasing reabsorption is manifested as an elimination of the high-energy edge of the spectrum - part where the absorption and emission bands overlap. In the spectral region where absorption is weak (the low-energy edge) the observed spectrum should be identical to the reabsorption free spectrum, i.e. first-generation spectrum. Applying normalization to the observed spectrum and appropriate scaling of the first-generation spectrum, it is possible to determine the value of reabsorption probability $r$ [22].

Fig. 2 shows the edge FL emission arising from an illumination spot far from the detection edge (reabsorption probability $46 \%$ ). The edge FL spectrum is plotted together with the first-generation FL spectrum and the filled area in between the curves represents the relative amount of reabsorbed photon flux.

Due to the long optical path lengths, the reabsorbed part of the edge fluorescent flux can be considered to be in thermal equilibrium with the emitting substance and can be described by the generalised Planck's law. The energy flux per unit energy interval of electro- or photoluminescent radiation emitted into a hemisphere is then given by [23]:

$$
F(T, E)=\frac{2 \pi}{h^{3} c^{2}} E^{3} \frac{1}{e^{\frac{\mu-E}{k_{B} T}}-1}
$$

where $h$ is the Planck's constant, $c$ is the speed of light, $k_{B}$ is the Boltzmann constant, and $E, \mu$ and $T$ are the photon energy, chemical potential and temperature, respectively. Equation (1) holds for objects with complete photon reabsorption / recycling. A multiplicative factor of $a$ has to be added to (1) for objects with emissivity (equal to absorptivity $a$ by Kirchoff's radiation law) less than one.

The reabsorbed part of the spectrum agrees well with expression (1) (Fig. 2), with an estimated temperature of $T=$ $360 \mathrm{~K}$, significantly higher than ambient temperature. The fit is valid within the spectral region where the absorption and emission bands of dye overlap and the reabsorption effect is the strongest (the high-energy edge).

To understand the origin of the high temperature photons observed we have analysed in detail the first-generation spectrum, focusing on the temperature effects, with the help of the Kennard-Stepanov theory. 


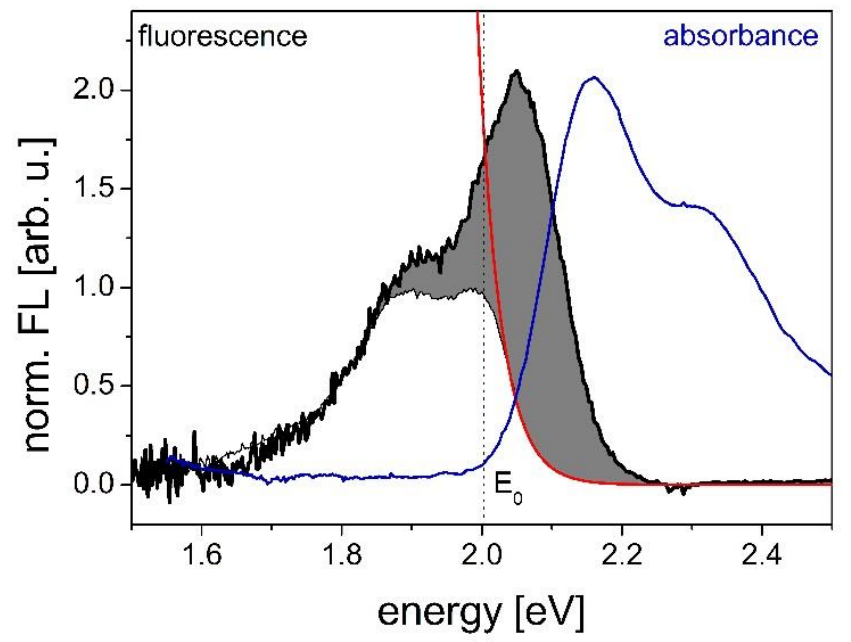

Fig. 2 Normalised reabsorbed FL spectrum (thin black line), scaled first-generation spectrum (thick black line), generalised Planck's law fit (red line) and absorbance spectrum of the dye (blue line). Filled area represents relative amount of reabsorption

The first-generation spectrum is shown in Fig. 3 (black line). In contrast to the reabsorbed spectrum (thin black line in Fig. 2), there is no spectral region that could be fitted by expression (1). This is due to the fact that photon emission by molecular systems in the absence of reabsorption is governed by the Kennard-Stepanov law (KS) $[17,18]$ which assumes a thermal equilibrium of vibrational levels of an excited state that act as a bath at temperature $T$ :

$$
\ln \left(\frac{c^{2} I(v)}{8 \pi h v^{3} \sigma_{a}(v)}\right)=-\frac{h v}{k_{B} T}+D(T)
$$
where $I$ is FL irradiance and $\sigma_{a}$ represents reabsorption crosssection. Equation (2) - which is the analogue of van Roosbroeck-Shockley relation for semiconductors, is an extension of Einstein's absorption and emission coefficients, neglecting stimulated emission.

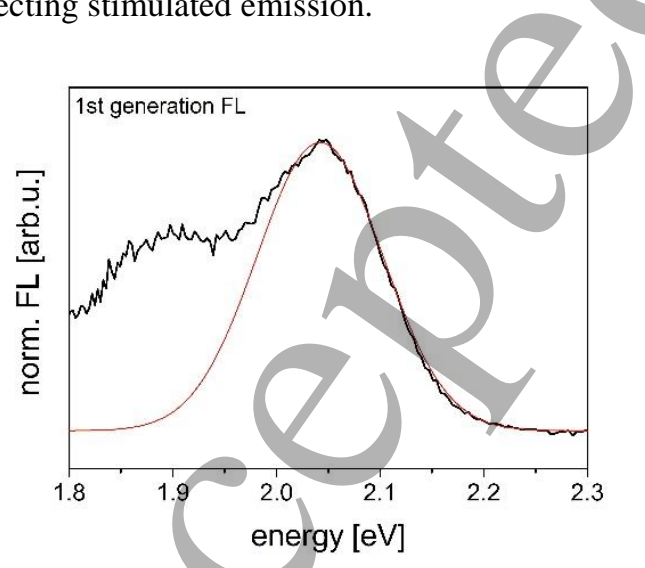

Fig. 3 The first-generation FL spectrum (black line) with the high energy band fitted by a Gaussian curve (red line) in accordance with Kazachenko's theory
A further tool in our analysis is offered by fitting the peaks in the spectra by Gaussian lines (red line in Fig. 3). A theory first proposed by Kazachenko [24] then gives a relationship between the Stokes shift $E_{S}$ and the width $\sigma$ of the Gaussian absorption / emission bands [24, 25]: $\sigma=\sqrt{E_{S} k_{B} T}$

The Gaussian fit in Fig. 3 is centered at $2.04 \mathrm{eV}$, in good agreement with the main band in the spectrum. The peak of absorbance was measured at $2.16 \mathrm{eV}$. Temperature extracted from the fit according to (3) then equals to $357 \mathrm{~K}$.

The dependence of temperature on the/reabsorption probability is plotted in Fig. 4. Determination of temperature is burdened by relatively large errors, and the uncertainty is larger for lower reabsorption. Despite the uncertainty in the extracted values we can conclude, after analysing all the measured excitation spots with various reabsorption levels, that the temperature of FL is higher than room temperature. It is worth noting that both the KS analysis of the first-generation spectrum and the generalized Planck's law applied to the reabsorbed spectra result in an elevated temperature. We shall also stress that all recorded spectra represent steady-state FL. FL time decay of our collectors span across the range of several nanoseconds/(see Appendix) indicating that the elevated temperature is preserved on this timescale.

Fig. 4 Dependence of the temperature of the hot FL flux on

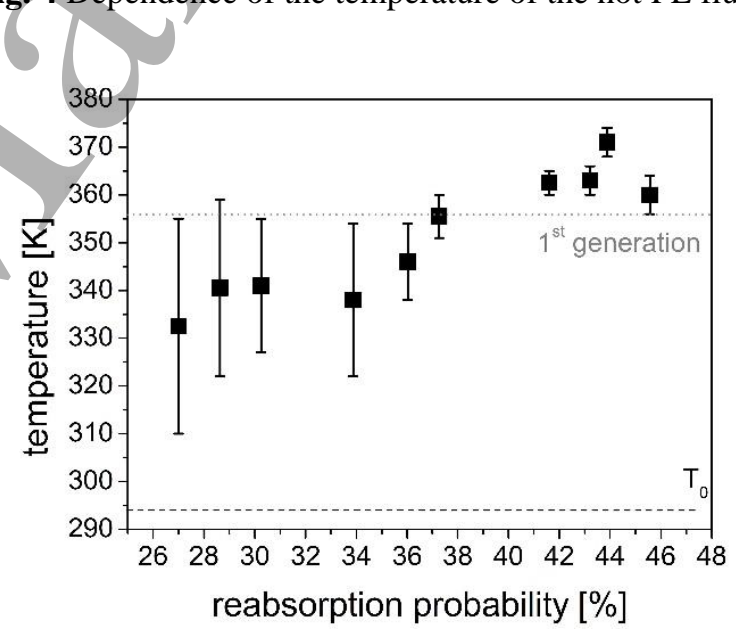

the reabsorption probability. Dashed black line - ambient temperature ( $294 \mathrm{~K})$, dashed gray line - temperature of the first-generation FL extracted from (3)

Although the Kennard-Stepanov-Kazachenko analysis of many dyes is in keeping with the conclusion that the vibrational subsystem is at the room temperature, there are frequent reports where the analysis produces elevated temperatures [19-21]. No general consensus appears to exist as to the origin of this phenomenon. Van Metter and Knox [20] suggest that high temperature of fluorescent flux can be extracted from KS relation plots in situations where vibrational equilibrium of molecules is not reached during the 
lifetime of their excited states. In such case, they conclude that two temperatures should be included in the KS formalism, representing both hot flux temperature and room temperature. However, this description is restricted to room temperature of ground state only, which might not be generally accurate.

Sawicki and Knox [21] analyse the spectra of alphaphycocyanin and report on the observation of hot FL and spectral dependence of temperature which consists of peaks reaching hundreds to thousands Kelvins. These complex temperature onsets are explained in terms of mixing of individual KS systems that originates in the presence of chemically different molecules or molecule manifolds.

Another correction to the KS relation was proposed by Mazurenko [26] for inhomogeneous broadening. He assumes a lack of orientational equilibrium of molecules before the emission of FL. Such a view could be applicable to our samples since we can expect discrepancies in molecules orientation due to interaction of dyes with solvent and polymer matrix.

\section{The potential of hot photons to increase solar cell efficiency}

Just as in the case of free charge carriers, hot photons represent a potential resource that could be used to increase the solar cell efficiency. A particularly promising aspect is that they are observed on timescales equal to the radiative lifetime (equivalent to open circuit of a solar cell) and can thus be considered as a means to increase the open circuit voltage.

To pave the way for a theoretical analysis of our results we summarise briefly the conclusions obtained by a thermodynamic analysis of PV conversion which will also determine the potential gains from tapping into the energy reservoir represented by the photons. Our view of PV conversion is summarised in Fig. 5.

The model considers incident photons above an energy threshold $E_{0}$ in a beam with étendue $\mathcal{E}_{i n}$ that are absorbed in an absorber. Photons which are not converted into electrical current are emitted, in a beam with étendue $\mathcal{E}_{\text {out }}$, in such a way that the photon numbers are conserved. We consider only the energy (rather than current) production which simplifies the discussion to an analysis at open circuit. The balance between photon absorption and emission, together with possible photon / carrier cooling by interaction with phonons, determines the thermodynamic parameters of photons in the absorber: energy per photon $u_{H}$, temperature $T_{H}$, entropy $s_{H}$ and chemical potential $\mu_{H}=u_{H}-T_{H} s_{H}$. The chemical potential $\mu_{H}$ is given by the approximate formula [27-29]:

$\mu_{H}=\left(1-\frac{T_{H}}{T_{S}}\right) E_{0}+k_{B} T_{H} \ln \left(\frac{T_{S}}{T_{H}}\right)-k_{B} T_{0} \ln \left(\frac{\varepsilon_{\text {out }}}{\varepsilon_{\text {in }}}\right)$

which can also been obtained directly from the ShockleyQueisser detailed balance [30]. For complete thermalisation when the temperature $T_{H}$ is equal to the ambient temperature $T_{0}, \mu_{H} \rightarrow \mu_{0}$ and Eq. (4) gives an expression for the open circuit voltage $V_{o c}$ of an ideal solar cell from $V_{o c}=\mu_{0} / q$. When photon temperature $T_{H}$ exceeds the ambient temperature $T_{o}$, photons collected in the absorber can, in principle, produce a higher voltage than generated by a standard solar cell.

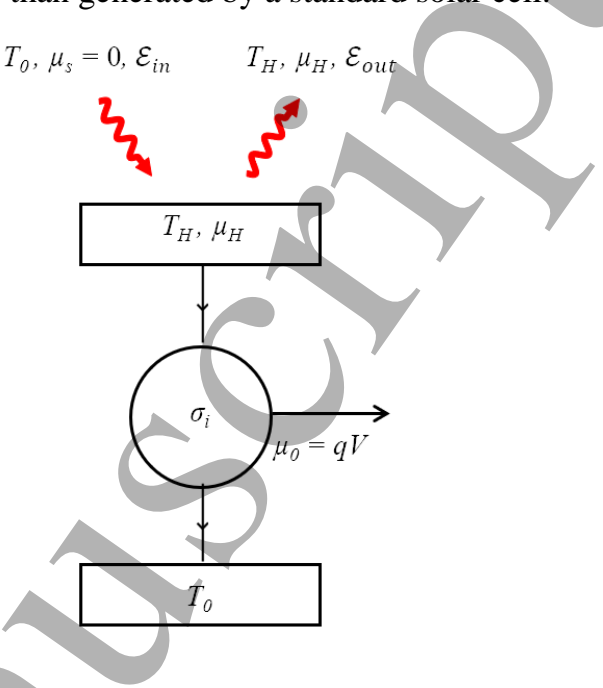

Fig. 5 A schematic view of the conversion process in hot carrier / photons solar cells. At open circuit, the thermodynamic parameters of the absorber are set by the balance between photon absorption and emission. A converter operating between the temperature $T_{H}$ of the absorber and the ambient temperature $T_{0}$ then absorbs heat equal to the photon energy $u_{H}$ from the absorber, rejects heat into the low temperature reservoir at temperature $T_{0}$, and produces work $\mu_{o}$. In an ideal converter, entropy $\sigma_{i}$ generated in the process is zero

In this paper we shall not specify the details of the conversion of photons from the absorber into work (or voltage) but consider an ideal converter that converts an amount of heat, equal to the energy $u_{H}$ of a photon, extracted from the absorber at temperature $T_{H}$ and cooled reversibly, without entropy generation $\left(\sigma_{i}=0\right)$, to the ambient temperature $T_{0}$. Details of the mathematics can be found, for example, in [29], with the result:

$\mu_{0}^{h o t}=u_{H}-T_{0} S_{H}=\left(1-\frac{T_{0}}{T_{H}}\right) u_{H}+\frac{T_{0}}{T_{H}} \mu_{H}$

In a more restricted setting, Eq. (5) has been obtained also by Ross and Nozik [1]. Using (4) and the approximate formula $u$ $=E_{g}+k_{B} T$ (see, for example, [31]), (5) can be transformed to:

$\mu_{0}^{\text {hot }}=\mu_{0}+q \Delta V_{\text {hot }}$

where:

$\Delta V_{h o t} \cong \frac{k_{B} T_{0}}{q}\left[\left(\frac{T_{H}}{T_{0}}-1\right)-\ln \left(\frac{T_{H}}{T_{0}}\right)\right]$ 
is the gain in open circuit voltage in hot carrier solar cell relative to conventional cells.

Values of $\mu_{H}$ extracted from the measured spectra and values of $\mu_{0}$ calculated according to (5) are plotted as a function of reabsorption in Fig. 6. Energy $E_{0}$ was assessed by using our "two-flux" model, where the photon flux emitted from the edge of the collector is divided into two fluxes - one completely reabsorbed and equilibrated, the other free of reabsorption. The division is given by intersection between first generation fluorescence spectrum and generalised Planck's law curve as shown on Fig. 2. A decrease of $\mu$ with increasing reabsorption reflects front surface escape losses that remove more photons with increasing optical path length.

The potential increase in voltage due to the high temperature photon flux, obtained from Eq. (7), is approximately $5 \mathrm{mV}$ - too small to be shown in Fig. 6. This small value is due to the fact that $\Delta V_{h o t}$ is proportional to $\left(T_{H^{-}}\right.$ $\left.T_{0}\right)^{2} / T_{0}^{2}$, and indicates that substantially higher hot carrier or photon temperatures are needed for a worthwhile improvement in the solar cell efficiency.

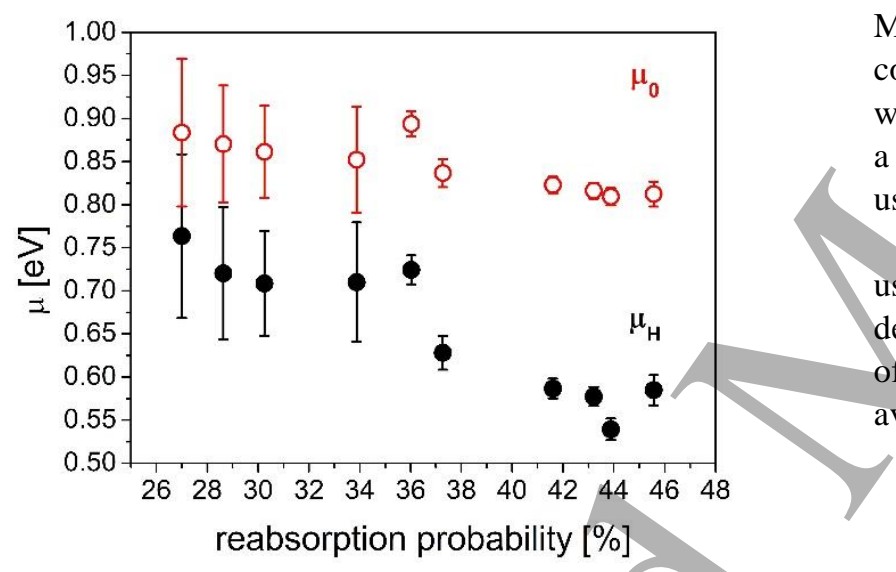

Fig. 6 Values of chemical potential plotted against reabsorption probability. $\mu_{H}$ represent values extracted from measured absolute spectra according (1) (full black circles). $\mu_{0}$ represent values obtained by an ideal room-temperature converter according (5) and (6) (red circles)

In conclusion, we showed that fluorescence spectroscopy is a useful tool for studying the performance of solar cell absorbers, as all the essential thermodynamic characteristics are imprinted in the light emitted from the absorber. This approach is also applicable for hot carrier-based devices since the presence of hot carriers is also reflected in the emitted flux, as previously observed by other workers [4, 7, 32]. Our observation of hot flux emitted by organic absorber and sustained over relatively long time (ns) is rather unique. By using a thermodynamic model of an ideal solar cell we calculated the enhancement of open-circuit voltage from the excess energy carried by hot particles. In the present case the enhancement is small due to the small difference between the hot photon and ambient temperatures. Notwithstanding, our method provides a robust framework to assess the potential of hot carriers - as manifested by the emission of hot photons with a simple expression for the maximum voltage enhancement.

\section{Acknowledgements}

Centre for Advanced Photovoltaics is supported by the Czech Ministry of Education, Youth and Sport. CZ.02.1.01/0.0/0.0/15_003/0000464.

\section{Appendix}

Fluorescent collectors were prepared by spin-coating a mixture of BASF perylene dye (Lumogen Red 305) with PMMA polymer on quartz glass plate. The PMMA was mixed in mass ratio 1:6 with dye solutions in toluene respectively. Collectors were excited by $446 \mathrm{~nm}$ laser with total incident power density approximately $40 \mathrm{~mW} / \mathrm{cm}^{3}$. The firstgeneration FL spectrum was obtained by collecting front surface emission of low-concentration dye sample $\left(5 \times 10^{-5} \mathrm{M}\right)$. Measurements of edge FL spectra were performed on highconcentration dye samples $\left(2 \times 10^{-4} \mathrm{M}\right)$, emission was collected with an integrating sphere and guided by optical fiber towards a Bentham spectrograph. The spectrograph was calibrated using a Bentham CL2 halogen spectral irradiance standard.

We have performed time-resolved FL measurement by using a Picoquant Fluotime FT200 system and recorded FL decays in timespan of $100 \mathrm{~ns}$ with 250 ps resolution. Example of FL decay at wavelength of $610 \mathrm{~nm}$ is shown in Fig. 1SI, the average time decay is $6 \mathrm{~ns}$.

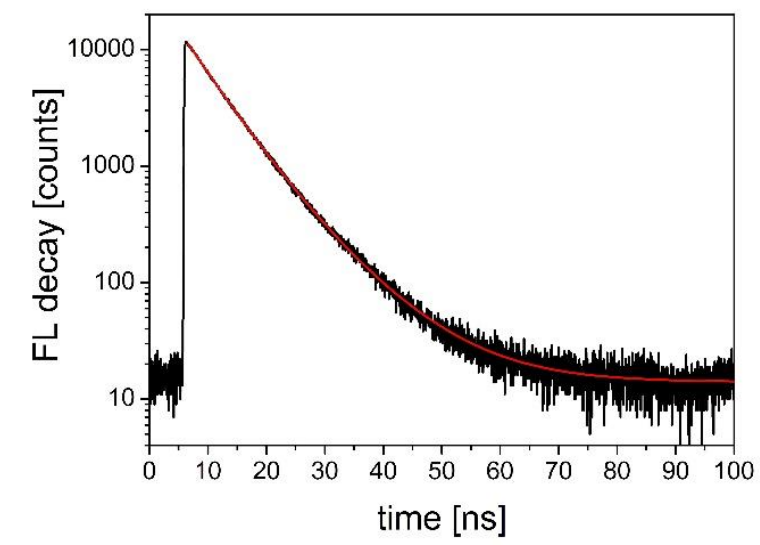

Appendix Fig. 1 FL time-decay measured at 610 nm, biexponential decay fit (red line)

\section{References}


[1] Ross, R.T. and A.J. Nozik, Journal of Applied Physics, 1982. 53(5): p. 3813-3818.

[2] Wu, J. and Z.M. Wang, Quantum dot solar cells. Lecture Notes in Nanoscale Science and Technology. 2014: Springer.

[3] Ridley, B., Journal of Physics C: Solid State Physics, 1982. 15(28): p. 5899.

[4] Shah, J. and R.C.C. Leite, Physical Review Letters, 1969. 22(24): p. 1304-1307.

[5] Le Bris, A. and J.-F. Guillemoles, Applied Physics Letters, 2010. 97(11): p. 113506.

[6] Rosenwaks, Y., et al., Physical Review B, 1993. 48(19): p. 14675-14678.

[7] Nozik, A., et al., Solid state communications, 1990. 75(4): p. 297-301.

[8] Conibeer, G., et al., Solar Energy Materials and Solar Cells, 2009. 93(6-7): p. 713-719.

[9] Jia, X., et al., Applied Physics Letters, 2018. 112(14): p. 143903.

[10] König, D., et al., Physica E: Low-dimensional Systems and Nanostructures, 2010. 42(10): p. 2862 2866.

[11] Würfel, P., Solar Energy Materials and Solar Cells, 1997. 46(1): p. 43-52.

[12] Tisdale, W.A., et al., Science, 2010. 328(5985): p. 1543-7.

[13] Kahmann, S. and M.A. Loi, Journal of Materials Chemistry C, 2019. 7(9): p. 2471-2486.

[14] Farrell, D.J., et al., Applied Physics Letters, 2011 99(11): p. 111102.

[15] Konovalov, I. and V. Emelianov, Energy Science \& Engineering, 2017. 5(3): p. 113-122.

[16] Rodière, J., et al., Applied Physics Letters, 2015 106(18): p. 183901.

[17] Kennard, E.H., Physical Review, 1918. 11(1): p. 29 38 .

[18] Stepanov, B.I., Sov. Phys. Doklady, 1957. 2: p. 81

[19] Knox, R., Acta Physica Polonica-Series A General Physics, 1999. 95(1): p. 85-104.

[20] Van Metter, R. and R. Knox, Chemical Physics, 1976. 12(3): p. 333-340.

[21] Sawicki, D.A. and R.S. Knox, Physical review A, 1996. 54(6): p. 4837.

[22] Kittidachachan, P., et al., CHIMIA International Journal for Chemistry, 2007. 61(12): p. 780-786.

[23] Wurfel, P., Journal of Physics C: Solid State Physics, 1982. 15(18): p. 3967.

[24] Kazachenko, L., Optics and Spectroscopy, 1965. 18: p. 397.

[25] Markvart, T. and R. Greef, J Chem Phys, 2004. 121(13): p. 6401-5.

[26] Mazurenko, Y.T., Optika i Spektroskopiya, 1972. 33(1): p. 42.

[27] Markvart, T., physica status solidi (a), 2008. 205(12): p. 2752-2756.

[28] Markvart, T., Applied Physics Letters, 2007. 91(6): p. 064102.
Markvart, T., Wiley Interdisciplinary Reviews: Energy and Environment, 2016. 5(5): p. 543-569.

Ruppel, W. and P. Wurfel, IEEE Transactions on Electron Devices, 1980. 27(4): p. 877-882.

Markvart, T., Journal of Optics A: pure and applied optics, 2007. 10(1): p. 015008.

Nguyen, D.-T., et al., Nature Energy, 2018. 3(3): p. 236-242. 